\title{
Center for Collective Usage "IKI-Monitoring" (Organization of Distributed Work with Extra Large Archives of Satellite Data for Solving Scientific and Applied Tasks)
}

\author{
Evgeny A. Loupian, Andrey A. Proshin, Ivan V. Balashov, Mikhail A. Burcev, \\ Alexandr V. Kashnitskiy, Vladimir A. Tolpin, Alexey A. Mazurov, Alexey M. Matveev, \\ Ivan A. Uvarov \\ Space Research Institute, Russian Academy of Sciences, Moscow, Russia \\ loupian@mail.ru
}

\begin{abstract}
Currently, Earth remote sensing data are widely used in solving a variety of scientific and applied problems related to monitoring the environment and natural resources. At the same time, solving many of them requires access to large multi-year archives of satellite data, as well as the availability of computing resources for processing and analyzing them. However, in the framework of individual projects, the creation of such often expensive infrastructure is not always possible. Thanks to the development of modern information technologies, this problem can be solved on the basis of the collective use of the infrastructure of the centers providing satellite data. For this reason in 2012 center for collective use "IKI-Monitoring" was launched by the Space Research Institute of Russian Academy of Sciences (IKI RAS). The paper is devoted to a brief description of the implementation of the center and its main features.
\end{abstract}

Keywords: remote sensing, satellite data, information systems, satellite data processing, remote analysis of satellite data, very big archives of satellite data 


\title{
ЦЕНТР КОЛЛЕКТИВНОГО ПОЛЬЗОВАНИЯ "ИКИ-МОНИТОРИНГ" (ОРГАНИЗАЦИЯ РАСПРЕДЕЛЕННОЙ РАБОТЫ СО СВЕРХБОЛЬШИМИ АРХИВАМИ СПУТНИКОВЫХ ДАННЫХ ДЛЯ РЕШЕНИЯ НАУЧНЫХ И ПРИКЛАДНЫХ ЗАДАЧ)
}

\author{
Е.А. Лупян, А.А. Прошин, И.В. Балашов, М.А. Буриеев, А.В. Кашницкий, В.А. Толпин, А.А. \\ Мазуров, А.М. Матвеев, И.А. Уваров
}

Институт космических исследований РАН, Москва, Россия

loupian@mail.ru

В настоящее время данные дистанционного зондирования Земли широко используются при решении самых разных научных и прикладных задач, связанных с мониторингом окружающей среды и природных ресурсов. При этом для решения многих из них требуется доступ к большим многолетним архивам спутниковых данных, а также наличие вычислительных ресурсов для их обработки и анализа. Однако, в рамках реализации отдельных проектов создание такой зачастую дорогостоящей инфраструктуры не всегда возможно. Благодаря развитию современных информационных технологий эта проблема может быть решена на основе коллективного использования инфраструктуры центров предоставления спутниковых данных. Именно с этой целью в 2012 году в ИКИ РАН был создан Центр коллективного пользования данными дистанционного зондирования ЦКП «ИКИ-Мониторинг». Настоящая статья посвящена краткому описанию реализации ЦКП и описанию основных его возможностей.

Ключевые слова: дистанционное зондирование, спутниковые данные, информационные системы, обработка спутниковых данных, удаленный анализ спутниковых данных, сверхбольшие архивы спутниковых данных

\section{Введение}

В последние десятилетия наблюдается стремительное развитие спутниковых систем дистанционного зондирования Земли. По состоянию на начало 2018 года на орбите Земли действовало более 400 космических аппаратов Д33, данные нескольких десятков из которых являются общедоступными. Одновременно с этим растет и качество данных, получаемых от современных систем Д33, которые в основном уже превратились из наблюдательных систем в измерительные. В результате, существенно возрос спрос на данные дистанционного зондирования Земли, которые в настоящее время используются для решения самых разных исследовательских и прикладных задач, связанных с мониторингом природной среды и антропогенных объектов. Существенно, что для решения многих из этих задач необходим доступ к большим многолетним архивам спутниковых данных и различным информационным 
продуктам, получаемым на их основе, а также значительные вычислительные ресурсы для их обработки и анализа. Однако, в рамках реализации отдельных тематических проектов далеко не всегда возможно создание зачастую дорогостоящей инфраструктуры для хранения и обработки больших массивов спутниковых данных.

Развитие современных информационных технологий позволило реализовывать принципиально новые подходы к организации работы со спутниковой информацией, обеспечивающие эффективное коллективное использование дорогостоящих вычислительных ресурсов центров сбора, обработки и представления данных дистанционного зондирования. Они позволяют не только обеспечить пользователям доступ к интересующим их спутниковым данным, но предоставить им разнообразные сервисы для их обработки и анализа на базе использования вычислительных ресурсов центров предоставления спутниковых данных. Именно на базе таких новых технологий и подходов в 2012 году был создан Центр коллективного пользования системами архивации, обработки и анализа данных спутниковых наблюдений ИКИ РАН - ЦКП «ИКИ-Мониторинг» [1]. Основной его целью является обеспечение распределенной работы со сверхбольшими архивами спутниковых данных в интересах различных научных и прикладных проектов.

В настоящей статье сначала приводятся интегральные характеристики реализованных архивов спутниковых данных не текущий момент времени, затем кратко рассматривается архитектура построения ЦКП «ИКИ-Мониторинг» и перечислены наиболее важные, на наш взгляд, технологические и программные решения. Далее следует краткое описание наиболее значимых информационных систем, реализованных на основе использования возможностей ЦКП. В заключение приводится наша оценка возможностей ЦКП «ИКИ-Мониторинг и обозначаются основные перспективы его развития.

\section{Архивы}

На текущий момент пользователям ЦКП "ИКИ-Мониторинг" доступны спутниковые данные, полученные более чем с 40 различных приборов, установленных как на российских, так и на зарубежных спутниках. Суммарный объем имеющихся в архивах данных превышает 2 Птб, а каждые сутки в архивы поступает около 2 Птб новых данных. При этом наиболее ранние данные в архиве датируются 1984 годом. Актуальная информация о наличии данных в архивах ЦКП «ИКИ-Мониторинг» может быть получена по ссылке http://ckp.geosmis.ru/default.aspx? page $=6$.

Одним из ключевых преимуществ ЦКП «ИКИ-Мониторинг» является уникальное для отечественных ресурсов временное и пространственное покрытие, которое в настоящее время составляет более $27 \%$ площади поверхности суши Земли. Благодаря возможности получения информации из единой системы работы с данными ФГБУ «НИЦ «Планета» [2] пользователи имеют возможность работы с глобальными покрытиями данных, получаемых различными российскими системами наблюдений. Доступ к этой информации организован в рамках соглашения Росгидромета и РАН, договора о сотрудничестве между ИКИ РАН и ФГБУ "НИЦ "Планета" об организации доступа к спутниковой информации для выполнения научных проектов и программ.

\section{Архитектура построения ЦКП «ИКИ-Мониторинг»}

Программно-аппаратный комплекс ЦКП «ИКИ-Мониторинг» построен на основе технологий и базового программного обеспечения, разработанных в отделе «Технологии спутникового мониторинга» ИКИ РАН [3]. Ключевым преимуществом используемых технологий является практически полная автоматизация всех процессов по сбору, архивации и обработке спутниковых данных. Общая архитектура построения ЦКП «ИКИ-Мониторинг» приведена на рис.1. 


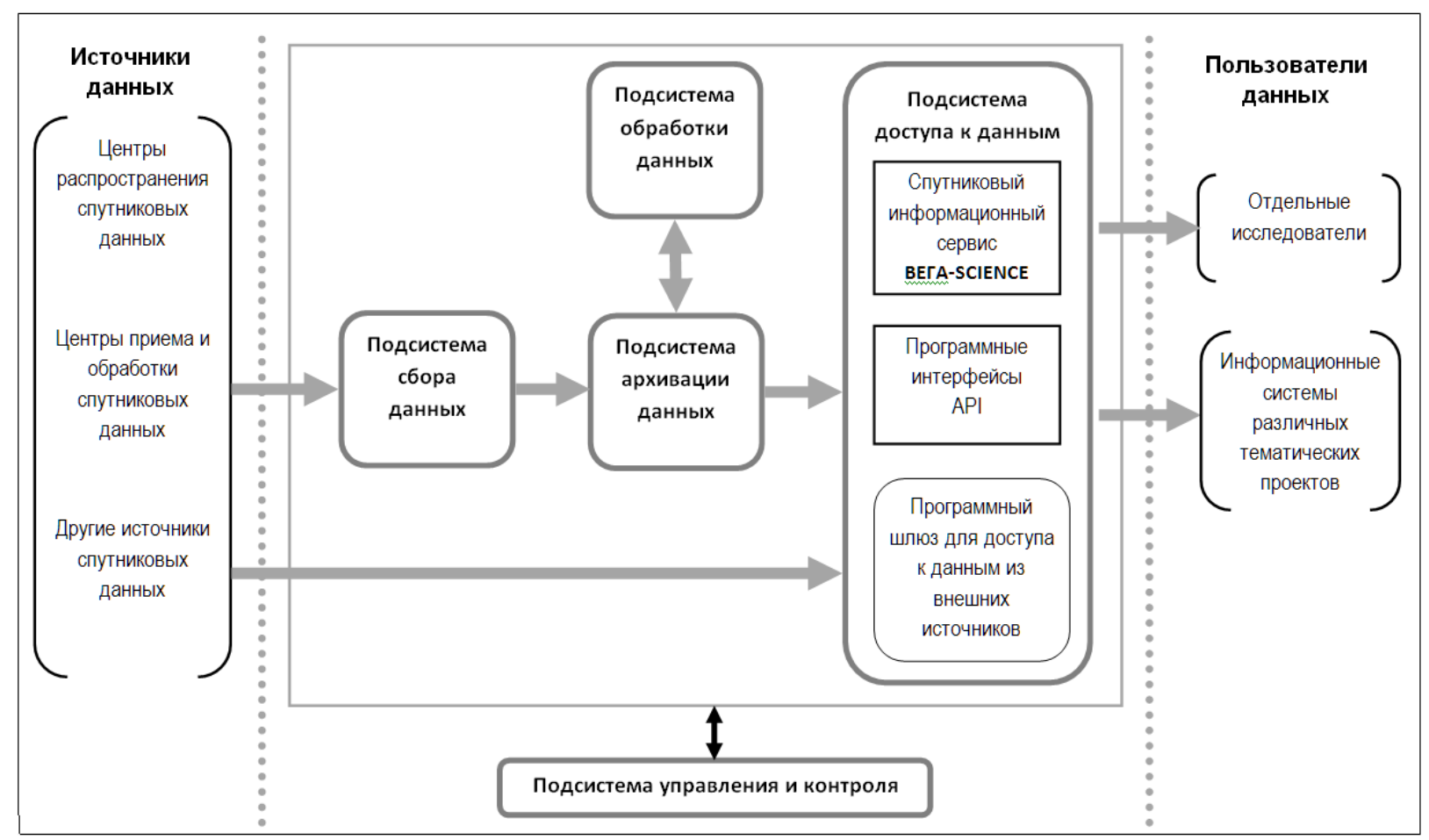

Рис. 1. Общая архитектура построения ЦКП «ИКИ-Мониторинг»

Подсистема сбора данных предназначена для получения спутниковых данных из различных источников и предварительной их подготовки для архивации или обработки. В качестве данных могут выступать как исходные спутниковые данные, так и различные информационные продукты, полученные на их основе.

Подсистема архивации данных отвечает за ведение сверхбольших распределенных архивов разнородных спутниковых данных и продуктов, полученных на основе их обработки. Она обеспечивает эффективный доступ к данным в архивах, а также позволяет предоставлять данные для последующей обработки.

Подсистема обработки данных предназначена как для проведения потоковой обработки поступающих в архивы оперативных данных, так и для построения различных информационных продуктов, получаемых на основе обработки уже имеющихся в архивах данных.

Подсистема обеспечения доступа к данным реализует три основных функциональных блока: спутниковый информационный сервис, программные интерфейсы для доступа к данным, их удаленной обработки и анализа, а также программный шлюз, предоставляющий доступ к данным, физически расположенным во внешних архивах спутниковых данных. Спутниковый информационный сервис «ВЕГА-SCIENCE» представляет из себя универсальный многофункциональный картографический интерфейс для доступа к данным и инструментам для их обработки и анализа в режиме, приближенном к реальному времени. На базе реализованных программных интерфейсов создаются тематические информационные системы, предназначенные для решения конкретных исследовательских и прикладных задач.

Подсистема управления и контроля предназначена для обеспечения бесперебойного функционирования центра коллективного пользования и управления всеми процессами по сбору, архивации и обработке спутниковых данных.

\section{Основные программные решения}

В реализации описанных выше программных подсистем используется большое число различных технологий и соответствующих им программных компонент, разработанных в отделе 
«Технологии спутникового мониторинга» ИКИ РАН за последние десятилетия. Ниже кратко описаны наиболее значимые из них.

Для построения унифицированных систем ведения сверхбольших распределенных архивов разнородных спутниковых данных используется технология UNISAT [4]. Ключевыми преимуществами этой технологии является поддержка механизма «виртуальных информационных продуктов», т.е. продуктов, которые динамически формируются по запросу пользователя на основе обработки имеющихся в архивах спутниковых данных, а также поддержка инструментов для удаленного анализа и обработки данных. Эти преимущества во многом достигаются благодаря использованию специальной стандартизованной базы данных, содержащей всю необходимую справочную информацию о спутниковых приборах наблюдения, имеющихся в архивах типах продуктов, а также о правилах построения «виртуальных» информационных продуктов. Специализированный служебный интерфейс позволяет с минимальными трудозатратами определять новые типы «виртуальных» продуктов, предназначенные для решения тех или иных научных или прикладных задач, после чего они становятся доступными пользователям без проведения какой-либо переобработки имеющихся в архивах данных.

Программная реализация подсистемы обработки данных основана на использовании разработанной в ИКИ РАН технологии распределенной многопотоковой обработки спутниковых данных [5] и соответствующего специализированного программного обеспечения, предназначенного для получения широкого спектра различных информационных продуктов, на основе исходных спутниковых данных и промежуточных результатов их обработки. Для обработки спутниковых данных также активно используется и различное свободно распространяемое программное обеспечения, в частности: GDAL, GRAS GIS, Proj, Imager и др. Реализованное программное обеспечение позволяет эффективно задействовать большой парк станций обработки спутниковых данных, на основе централизованной диспетчеризации потоков данных и управления всеми задачами по обработке спутниковых данных.

Для построения специализированных картографических интерфейсов применяется разработанная в ИКИ РAH технология GEOSMIS [6]. Данная технология рассчитана на создание интерфейсов работы с большими распределенными многомерными архивами спутниковых данных и результатов их обработки. Ключевым ее преимуществом является поддержка инструментов для удаленного анализа и обработки спутниковых данных. Разработанная технология позволяет с минимальными трудозатратами реализовывать новые тематические интерфейсы для решения как научных, так и прикладных задач. Благодаря использованию двухуровневой системы тематических вкладок, удается достичь высокой гибкости и четкой структурированности создаваемых интерфейсов. Важно отметить, что создание подобных интерфейсов стало возможным благодаря стремительному развитию информационных технологий, и, в частности, технологий, позволяющих в рамках WEB интерфейсов реализовывать сложный функционал, который раньше был доступен только в настольном программном обеспечении. С этой целью в ИКИ РАН была разработана технология создания инструментов для удаленного анализа и обработки спутниковой информации, находящейся в сверхбольших распределенных архивах спутниковых данных [7].

Для решения широкого спектра задач, связанных с поддержкой бесперебойной работы сложных программно-аппаратных комплексов в ИКИ РАН была разработана система документирования и контроля (СДКП) [8]. Ключевым ее элементом является система ведения сбоев, позволяющая автоматически детектировать большое число различных типов неполадок и сбоев в работе комплекса, оперативно оповещать о них персонал и отслеживать процесс их устранения. 


\section{Реализованные информационные системы}

В настоящее время на основе возможностей предоставляемых центром коллективного пользования ЦКП «ИКИ-Мониторинг» функционирует более 12 различных информационных систем научного назначения и, частности:

- Информационная система "Аврора-Арктика" (http://dev.aurora.geosmis.ru/), разработанная в ИКИ РАН и введенная в опытную эксплуатацию в 2017 году. Система ориентирована на работу данными наблюдений ионосферы Земли, полученных со спутников серии DMSP, показателей наблюдения солнечного ветра по данным NOAA, метеорологических данных, а также данных спутниковых наблюдений в оптическом диапазоне, полученных различными системами (MODIS, AVHRR и др.) и прогностической информацией о границах аврорального овала.

- Информационная система «Дистанционный мониторинг активности вулканов Камчатки и Курил» VolSatView (http://volcanoes.smislab.ru/) [9]. Основной задачей системы является обеспечение специалистов-вулканологов оперативными спутниковыми данными и различными информационными продуктами, получаемыми на основе их обработки, для мониторинга и изучения вулканической активности Камчатки и Курил. Работам по этой тематике посвящен п. 3.2.2 настоящего отчета.

- Спутниковый сервис See The Sea (STS, http://ocean.smislab.ru/) [10] - информационная система, ориентированная на работу с данными спутниковых наблюдений для решения междисциплинарных задач исследования Мирового океана. Особое внимание в системе уделяется возможностям работы с данными спутниковой радиолокации. Система призвана обеспечить специалистам, работающим в области исследования Мирового океана, возможность одновременной работы с различными видами спутниковой информации и удобный инструментарий, позволяющий проводить ее комплексный анализ.

- ИС Аналитик ДЗЗ ЕФИС ЗСН «ИКИ-Мониторинг» является частью Единой информационной системы земель сельскохозяйственного назначения (ЕФИС ЗСН, http://efis.geosmis.ru/). ЕФИС ЗСН предназначена для обеспечения Минсельхоза РФ и подведомственных ему учреждений и организаций актуальной и достоверной информацией о землях сельскохозяйственного назначения и землях, используемых или предоставленных для ведения сельского хозяйства в составе земель иных категорий, в том числе данными о местоположении, состоянии и фактическом использовании таких земель и состоянии сельскохозяйственной растительности. Аналитик ДЗЗ ЕФИС ЗСН разрабатывался и развивается ООО "ИКИЗ", ИКИ РАН, ООО «АнТ», Аналитический центра МСХ РФ.

ЦКП «ИКИ-Мониторинг» также используется для проведения разработок методов обработки и анализа спутниковых данных, многие из которых впоследствии внедряются в различные прикладные информационные системы дистанционного мониторинга, некоторые из которых перечислены ниже:

- Информационная система дистанционного мониторинга Федерального агентства лесного хозяйства ИСДМ-Рослесхоз (http://www.pushkino.aviales.ru) [11], предназначенная для сбора информации о пожарах по всей территории России, сбора информации о состоянии окружающей среды, подготовки информационных продуктов для анализа пожарной обстановки и последствий пожаров и оперативного распространения накопленной информации.

- Отраслевая информационная система мониторинга Федерального агентства рыбного хозяйства (ОСМ Росрыболовства) (http://osm.smislab.ru/), ориентированная на получение информации о деятельности промысловых судов. В настоящее время ОСМ обеспечивает мониторинг российских и иностранных судов, ведущих промысел в экономической зоне России, а также российских судов, находящихся на промысле в различных районах мирового океана. ОСМ обеспечивает контроль нескольких тысяч судов, из которых около 
2000 судов обычно находятся на промысле. По числу судов, находящихся под контролем, система является самой крупной в мире.

- Информационная система «Вега-Агрометеоролог» (http://agrometeo.geosmis.ru), предназначенная для комплексного агрометеорологического мониторинга, которая разрабатывается ИКИ РАН совместно с Гидрометцентром России, ФГБУ «НИЦ «Планета» и ВНИИСХМ. Система предназначена для обеспечения экспертов инструментами совместного анализа регулярной спутниковой информации и данных наземных наблюдений по всей территории сельскохозяйственных земель России с учетом агроклиматического районирования.

\section{Заключение}

ЦКП «ИКИ-Мониторинг» предоставляет уникальные возможности для эффективной работы с данными дистанционного мониторинга Земли для решения различных научных и исследовательских задач, позволяя избежать необходимости создания дорогостоящей инфраструктуры для каждого из проектов. Возможностями ЦКП «ИКИ-Мониторинг» сейчас пользуются более 70 различных научных организаций, что позволило реализовать целый ряд различных научных информационных систем. По объемам данных, находящимся в onlineдоступе, ЦКП «ИКИ-Мониторинг» входит сегодня в первую десятку мировых центров предоставления доступа к информации дистанционного мониторинга. По реализованному функционалу, обеспечивающему распределенный анализ и обработку данных, он является ведущим центром в России и конкурирует с наиболее передовыми мировыми системами этого класса.

В перспективе, в первую очередь, планируется продолжить работы по совершенствованию и расширению предоставляемого пользователям функционала для анализа и обработки спутниковых данных в режиме, приближенном к реальному времени.

Работы выполнялись в рамках темы «Мониторинг» (госрегистрация № 01.20.0.2.00164).

\section{References}

[1] Loupian E.A., Proshin A.A., Burtsev M.A., Balashov I.V., Bartalev S.A., Efremov V.Yu., Kashnitskiy A.V., Mazurov A.A., Matveev A.M., Sudneva O.A., Sychugov I.G., Tolpin V.A., Uvarov I.A. IKI center for collective use of satellite data archiving,processing and analysis systems aimed at solving the problems of environmental study and monitoring, Sovremennye problemy distantsionnogo zondirovaniya Zemli iz kosmosa, 2015, Vol. 12, No 5, pp. 263-284.

[2] Loupian E.A., Milexin O.E., Antonov V.N., Kramareva L.S., Bourtsev M.A., Balashov I.V., Tolpin V.A., Solovyev V.I. System of operation of joint information resources based on satellite data in the Planeta Research Centers for Space Hydrometeorology, Russian Meteorology and Hydrology, 2014, Vol. 39, Issue 12, pp. 847-853. DOI: 10.3103/S1068373914120103.

[3] Loupian E.A., Balashov I.V., Bourtsev M.A., Efremov V. Yu., Kashnitskiy A.V., Kobets D.A., Krasheninnikova Yu. S., Mazurov A.A., Nazipov R.R., Proshin A.A., Sychugov I.G., Tolpin V.A., Uvarov I.A., Flitman E.V. Development of information systems design technologies, Sovremennye problemy distantsionnogo zondirovaniya Zemli iz kosmosa, 2015, Vol.12, No 5, pp. 53-75.

[4] Proshin A.A., Loupian E.A., Balashov I.V., Kashnitskiy A.V., Bourtsev M.A. Unified satellite data archive management platform for remote monitoring systems development, Sovremennye problemy distantsionnogo zondirovaniya Zemli iz kosmosa, 2016, Vol. 13, No. 3, pp. 9-27. DOI: 10.21046/20707401-2016-13-3-9-27.

[5] Kobets D.A., Matveev A.M., Mazurov A.A., Proshin A.A. Organization of automated multithreaded processing of satellite information in remote monitoring systems, Sovremennye problemy distantsionnogo zondirovaniya Zemli iz kosmosa, 2015, Vol.12, No 1, pp. 145-155. 
[6] Tolpin V.A., Balashov I.V., Efremov V. Yu., Loupian E.A., Proshin A.A., Uvarov I.A., Flitman E.V. The GEOSMIS System: Developing Interfaces to Operate Data in Modern Remote Monitoring Systems, Sovremennye problemy distantsionnogo zondirovaniya Zemli iz kosmosa, 2011, V. 8, No 3, pp. 93-108.

[7] Kashnitskiy A. V., I. V. Balashov, E. A. Loupian, V. A. Tolpin, and I. A. Uvarov, Development of software tools for the satellite data remote processing in contemporary information systems, Sovremennye problemy distantsionnogo zondirovaniya Zemli iz kosmosa, 2015, Vol 12, No 1, pp. 156-170.

[8] Balashov I.V., Efremov V. Yu., Mazurov A.A., Mamaev A.S., Matveev A.M., Proshin A.A. Features of Remote Monitoring Distributed Systems Control and Monitoring, Sovremennye problemy distantsionnogo zondirovaniya Zemli iz kosmosa, 2011, V. 8, No 3, pp. 161-166.

[9] Efremov V.Yu., Girina O.A., Kramareva L.S., Loupian E.A., Manevich A.G., Melnikov D.V., Matveev A.M., Proshin A.A., Sorokin A.A., Flitman E.V. Creating an information service «Remote Monitoring of Active Volcanoes of Kamchatka and the Kuril Islands», Sovremennye problemy distantsionnogo zondirovaniya Zemli iz kosmosa, 2012, Vol. 9, No. 5, pp. 155-170.

[10] Lavrova O. Yu., Loupian E.A., Mityagina M.I., Uvarov I.A., Bocharova T. Yu. See the Sea - Multi-User Information System Ocean Processes Investigations Based on Satellite Remote Sensing Data, Bollettino di Geofisica teorica ed applicata. An International Journal of Earth Sciences, 2013, V.54, pp. 146-147.

[11] Loupian E.A., Bartalev S.A., Ershov D.V., Kotelnikov R.V., Balashov I.V., Bourtsev M.A., Egorov V.A., Efremov V. Yu., Zharko V.O., Kovganenko K.A., Kolbudaev P.A., Krasheninnikova Yu. S., Proshin A.A., Mazurov A.A., Uvarov I.A., Stytsenko F.V., Sychugov I.G., Flitman E.V., Khvostikov S.A., Shulyak P.P. Satellite data processing management in Forest Fires Remote Monitoring Information System (ISDM-Rosleskhoz) of the Federal Agency for Forestry, Sovremennye problemy distantsionnogo zondirovaniya Zemli iz kosmosa, 2015, Vol.12, No 5, pp. 222-250. 